\title{
Application of Data and Information Visualization in Electronic Commerce
}

\author{
Huan Huang ${ }^{1}$ \\ Guangdong Polytechnical Normal University \\ Guangzhou, 510665, China \\ Email: $434424462 @ q q . c o m$ \\ Jiandong Zhao ${ }^{2}$ \\ Guangdong Polytechnical Normal University \\ Guangzhou, 510665, China \\ E-mail: 910465692@qq.com

\section{Zhichao $\mathbf{X u}^{3}$} \\ Guangdong Polytechnical Normal University \\ Guangzhou, 510665, China \\ E-mail: $576726974 @ q q . c o m$
}

In order to promote the application of science visualization technology in the e-commerce industry, the paper investigated the utilization of massive data and information through visual analysis to explore the hidden links between data and information. By taking Taobao website as an example, this article chooses to improve the product display page and the product evaluation page. Data mining is used to find the hidden information between data and information. Through data analysis, we can find the connection between hidden information. Based on improvement of the display page and the product evaluation page, we can display the hidden information of the system with visual charts to improve the sales volume of the products. For the innovation point, this paper has found that the addition of favourable rate and age-appropriate data to the display page can attract the users' attention and promote the increase of page views. In the commodity evaluation page, the key words and keywords in the evaluation can improve the consumers' desire to buy. By applying data and information visualization techniques to e-commerce, the hidden connections between data and information can facilitate the development of e-commerce.

ISCC2017

16-17 December 2017

Guangzhou, China

\section{${ }^{1}$ Speaker}

This work was supported by the Science and Technology Planning Project of Guangdong Province(Grant No.2016A040404016), the special innovation project of Educational Commission of Guangdong Province(Grant No.2014WTSCX078) and Ebusiness innovation and entrepreneurship course project of Guangdong.

${ }^{2}$ Speaker

${ }^{3}$ Speaker 


\section{Introduction}

At present, less scholars have studied the application of data and information visualization in e-commerce. For example, R.Wang and R.C.Li in the article " Application of Information Visualization in the Store Design of Taobao" only talked about the information visualization design can bring about good experience to the users.[1]But now, the e-commerce industry is developing well with various e-commerce sites emerging. How to stand out among the many e-commerce sites is the top priority that we have to consider. As Yang Feng in his article "Analysis of Information Visualization in the E-commerce Application Status", the article analyzed the types of information in e-commerce. By classfying the information, it can be pointed out that information visualization can be applied to the management of the information to improve the website efficiency; however, Yang Feng only discussed some simple application of information visualization in the e-commerce system, [2] but few scholars have noticed the hidden messages in data and information, even these hidden messages sometimes play a decisive role. This paper is, based on the analysis of hidden information in the data, is to find out the links between them to promote the development of the e-commerce industry.

With the development of science and technology, there are tons of data and information that people available every day. How to organize these data and information is one of the most difficult problems. In order to solve this problem, the data visualization technology has been greatly developed and supported. The data and information visualization technology is a relatively broad field applied to geography, meteorology, medicine and other fields. And using data and information visualization technology in business has brought about unlimited business opportunities.[3] How to use information visualization technology to find hidden information in data to promote the development of e-commerce is the focus of this paper.

\section{Visualization}

Data visualization and information visualization are both visualizations. The data visualization represents each data item in the database as a single image element, and a large number of data set constitute the data image. At the same time, each attribute value of the data is represented in the form of multidimensional data, which can be viewed from different dimensions to further observe and analyze the data. The information visualization, a combination of data and design of the picture, is intending to visualize the data in a visual way. . It can help individuals or organizations communicate information to the audience in a short and effective manner. [4]

\section{Visual Objects in E-commerce}

From a management information system perspective, a complete e-commerce system must contain a complete information system. The information is stored in the information system in the form of data. The electronic commerce system mainly has the following kinds of information: user information, commodity information, merchant information, purchase information, supply information and evaluation information, etc.. Through the processing and analysis of these information, we can strengthen the consolidation and integration of data information so as to strengthen the e-commerce system management model and improve the work efficiency. [5] 


\section{Theoretical Foundations of Data and Information Visualization}

The theoretical basis of data and information visualization mainly refers to the modern visualn theory based on IT technology. The theories used in e-commerce systems include: the visual management theory characterized by glasnost and visual display; a kanban management theory that carries out logistics or information flow. By collecting, sorting and analyzing data, we can reach the essence of the object and predict the future statistical theory of the object. The logic theory of the logical structure of the information is divided into the logic of the time, space and quantity of the data generated. By studying these theories, we can organize the information more comprehensively and make clear the logical relation between information. Then we find out the hidden message between the data and the information. Finally, we can use the chart to show the hidden information so that people can see and understand with the naked eye.

\section{Key Technologies for Data and Information Visualization}

Data and information visualization technology refers to a visual interface through humancomputer interaction. Data and information visualization technology is an organic combination of data mining, graphics processing, scientific calculation and other technologies. The data mining technology extracts hidden information and knowledge unknown but potentially useful in a large number of incomplete, fuzzy and random real data. The technology of scientific computing refers to the mathematical calculation encountered in the processing of scientific research and engineering. The graphic processing technology refers to the graphical representation of the potential useful information and knowledge in the data mining techniques to facilitate the intuitive understanding of the data. [6]

\section{Application of Data and Information Visualization in E-commerce System}

Data and information visualization technology has many applications in e-commerce, such as information visualization of goods, information visualization of transaction process, etc. Large data analysis found that: the main factor that customers consider when buying goods is a course of commodity evaluation which evaluates the user of goods as purchased. On different websites, the commodity evaluation shows in a different way. In Taobao, the evaluation of goods can be divided into high praise, middle evaluation and poor evaluation. On Jingdong online, the evaluation of commodities is replaced by stars, which is less valuable than the direct division of ratings in Taobao. As everyone's positioning of the star is different, it may result in deviation. On vip.com, the commodity evaluation conforms to the star standard. By contrast, Taobao's high praise, middle evaluation and poor evaluation can be more intuitive to allow users to understand the goods [7].

Many e-commerce sites now have additional evaluation functions. Additional evaluation refers to the evaluation of the goods as purchased by the purchasers after the use of the commodity for a period of time. The additional evaluation can reflect the real quality, value and other data of the commodity[8]. Through big data analysis, most people look for additional comments when they buy a product; however, the current major e-commerce system's additional comment system can only be published. Without analyzing the data and information of the additional comments, the user can not directly understand whether the additional comments are good or bad. Users have to browse every additional comment. This is a waste of time and is not conducive to promote consumption. Based on the above reasons, this paper tries 
to improve the information of commodity interface and commodity evaluation page. We use data and information visualization technology to facilitate the development of the current ecommerce industry.

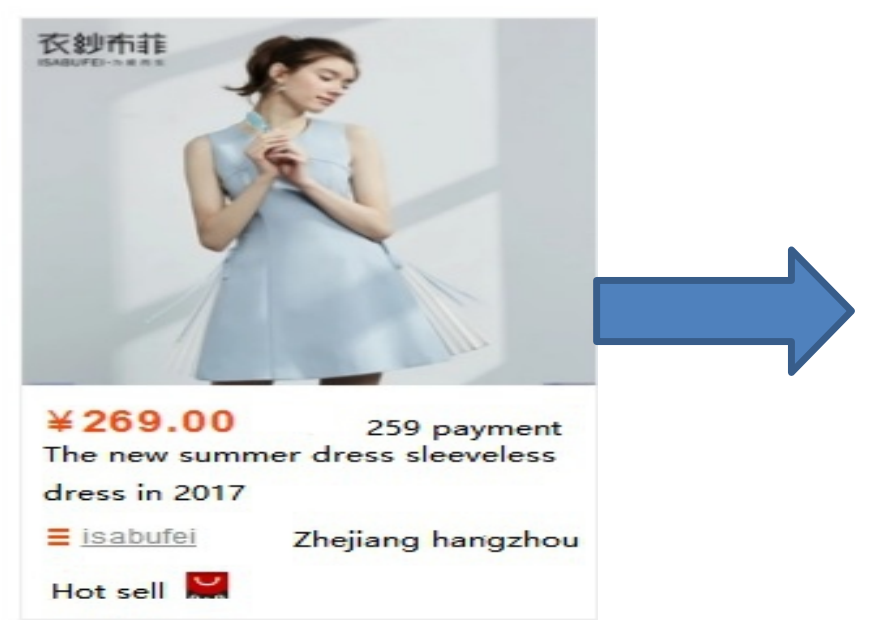

Figure 1 : Traditional Commodity Interface

Figure 2 : Improved Product Interface

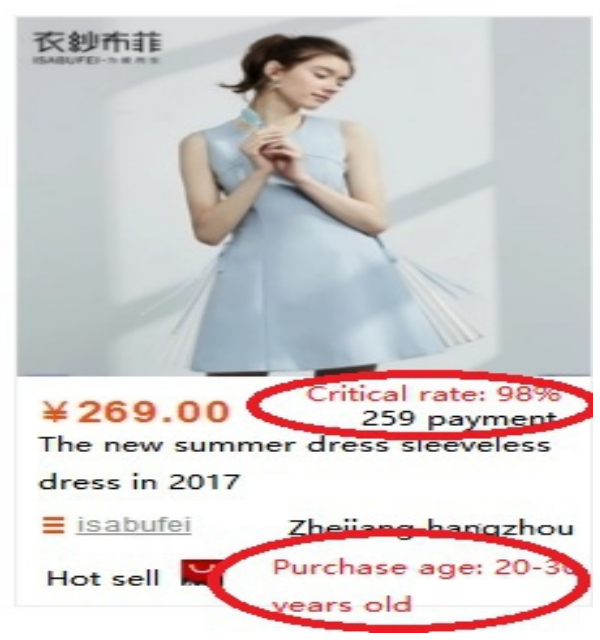

Figure 1 shows the application of the visual technology of commodity information in the traditional e-commerce system. Figure 1 shows the sample of the goods, the price, whether it is the new product, whether it is the hot seller, the number of payment, the name of the goods, the name of the store, the delivery place and whether the customer is online, etc.; however, taobao has developed rapidly. The increase of taobao merchants has led to a large number of commodities, as a result, the price gap and age range have been increasing. How to find a suitable commodity in a mass of commodities is a top priority for users.

Figure 2 shows a number of additional information on the display page intending to address the problems available on the traditional e-commerce sites. We use data mining technology to realize the innovation of information visualization. The information displayed on the display interface should be information that can determine the user's decision. In addition, since there are so many products, the users can only judge whether they are suitable for themselves; therefore, the author makes a statistical analysis of the age of the purchaser to obtain an interval value of an age. The author then adds information to the product display page for user reference. 


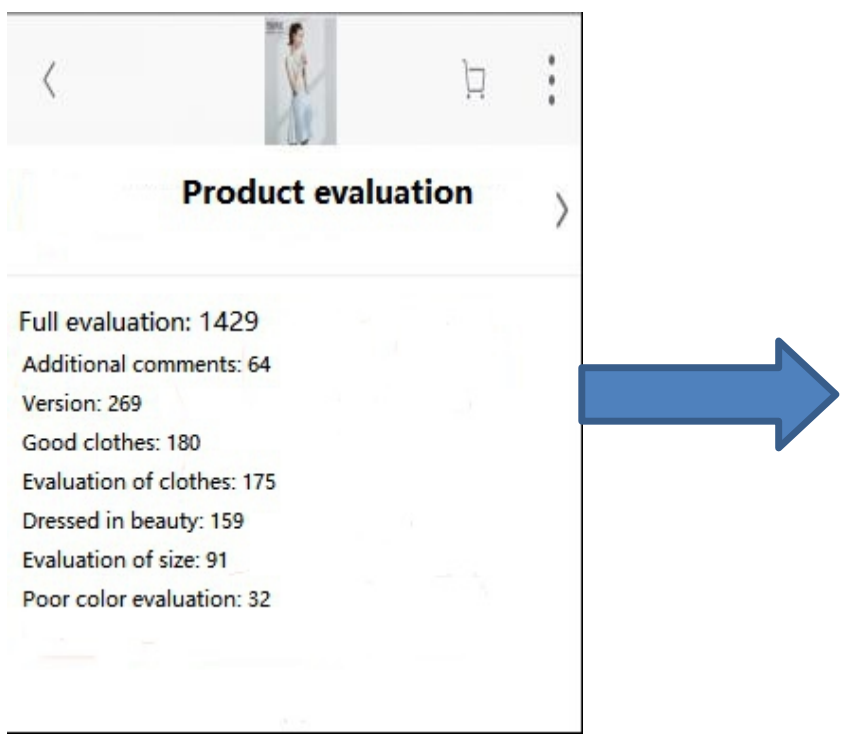

Figure 3 : Traditional Evaluation Page

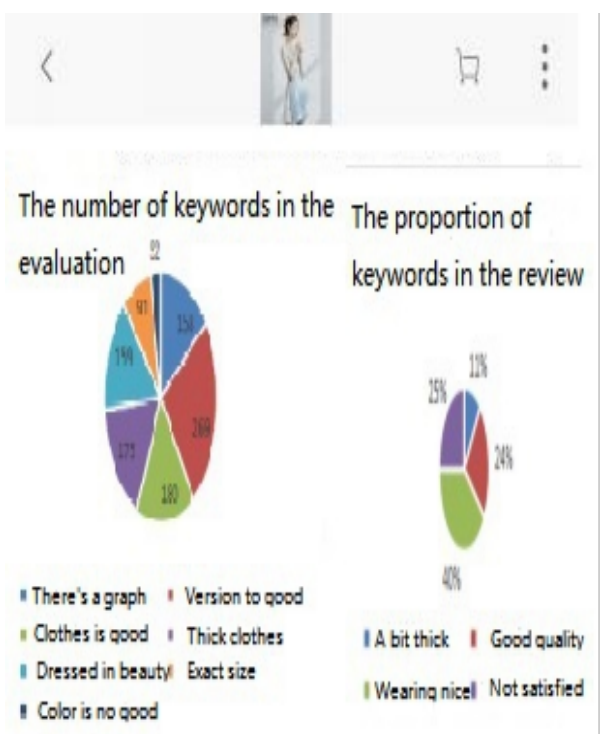

Figure 4 : Improved Evaluation Page

Figure 3 is the traditional product evaluation page in e-commerce, which is displayed by the keyword display method through data mining. There is no correlation between the data, and there is no indication that the data of each keyword is the proportion of the total data.

Figure 4 shows that we use data mining and analysis techniques to systematically analyze the data and information in e-commerce, and visualize the keywords with pie charts. It can clearly show the proportion of different keywords in all keywords so that users can see the proportion of keywords at a glance. By graphing data information, it becomes the user visual information. As different users have different needs, we can better meet the users' demand by using pie chart to show the proportion of keywords. For example, $32 \%$ of the people in the picture think the shape of the product is good, so the data is valuable for the customer who is required for the type version. The users with different requirements can refer to different data accounts. Additional comments are important factors when choosing a product. Additional comments are the information of the user's experience, which is more valuable. Therefore, the 
keyword information in the supplemental comment is statistically analyzed, then the pie chart of the additional comment keyword is made. In this picture, it is clear that $25 \%$ of users are not satisfied with the goods, which is a data worthy of reference and can play a decisive role in the purchase of goods by users.

\section{The Experimental Conclusion}

Due to time limit, we chose to release a questionnaire on electronic business innovation to verify whether the improvement of the product display page and the product evaluation page is in line with consumers' consumption psychology. The questionnaire results are shown in Figure 5 below:

\section{The results of the questionnaire survey}

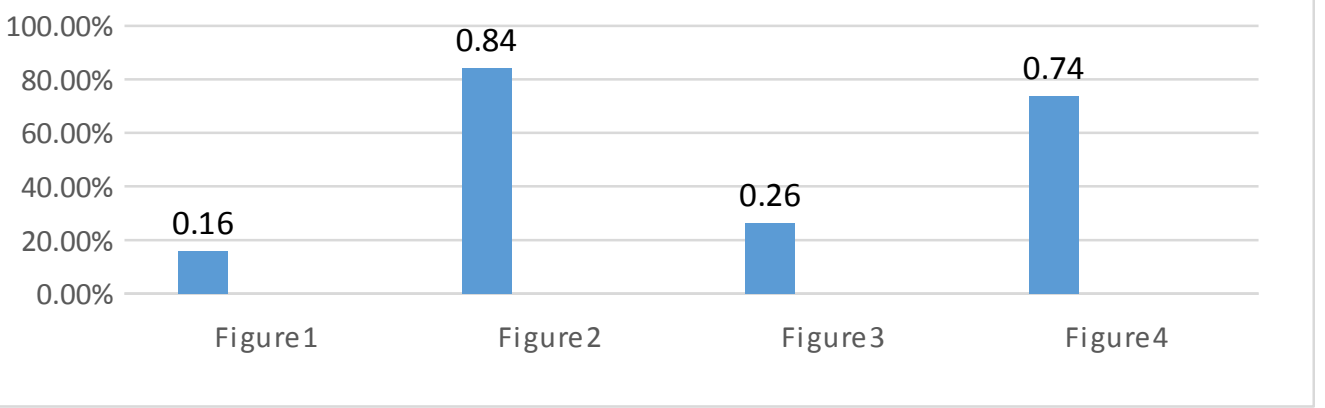

\section{Figure 5: Resultes of Improved Questionnaire Survey}

According to the questionnaire results, $84.21 \%$ and $73.69 \%$ respondents chose the improved product display page and the product evaluation page. Conclusion 1: if we add a good rating and age information to the display page, it will attract more attention than traditional pages. Conclusion 2: if the proportion of keywords and the proportion of supplementary comments are added to the product evaluation page, it is more consistent with the user's demand for product information. The users can select goods according to their own needs, which can promote the sales of goods. In the next study, we will select ourselves to design an e-commerce site and operate online to verify the improved page of the author can improve product sales and promote the development of e-commerce industry in the actual sales of the same product.

\section{Summary}

In this paper, the data and information of traditional e-commerce websites are excavated to find the hidden information between the data. Analysis of the information reveals that these hidden messages are not independent but related mutually. The links between the hidden messages are displayed in a graphical manner. According to the statistics, the charts are improved on the traditional display page and the product evaluation page. Finally, it is found that the improved page is more in line with the user's demand, which is beneficial to save the users' time and increase the sales volume of the goods, thus promoting the development of the ecommerce industry.

At present, data and information visualization have been widely used in many industries. And there are also many applications in the e-commerce industry, too. Due to various product information in the e-commerce, how to use the way of data and information visualization to display the useful information to consumers directly so as to promote consumption and improve the operating rate is considered in this paper. The design of this paper uses data and information 
visualization technology at the base level for the purpose of providing reference for future information visualization in the e-commerce industry.

\section{References}

[1]R.Wang,R.C.Li. The Application Of Information Visualization In The Store Design Of Taobao [D].Art Education Research.2015:70-71. (In Chinese)

[2]F.Yang. Analysis Of Information Visualization Application In E-commerce [J]. Information Systems, 2007,03: 412-415. (In Chinese)

[3] X.L.DU,Y.M.WEI,L.D.WU. Interactive Details on Demand Visual Analysis on Large Attributed Networks[J/OL]. Chinese Journal of Electronics,:1-10(2017-10-31).

[4]Y.Gao. Research And Analysis Of Information Visualization Technology[J]. Science And Technology Innovation And Application, 2016,01: 59-63. (In Chinese)

[5]F.Liu .The Technology And Application Research In Information Visualization [D]. Zhejiang University, 2013. (In Chinese)

[6]P.Pan. The Application Of Information Visualization In E-commerce [J]. The Application Of Science And Technology2011(22):89. (In Chinese)

[7]Y.B.Yang,B. Liu,Y.M. Qi. Summary Of Information Visualization Research [J/OL]. Journal Of Hebei University Of Science and Technology, 2014,35 (01): 91-102. (In Chinese)

[8]X.P.Xu,X.M.Wang,R.Y.Peng,D.W.Wang. Basic Principles And Applications Of Information Visualization [J]. Chinese Stereology And limage Analysis, 2007,12,01:75-78. (In Chinese) 